\title{
Electrochemical ascorbic acid sensor based on DMF-exfoliated graphene $\dagger$
}

\author{
Gareth P. Keeley, ${ }^{a b}$ Arlene O'Neill, ${ }^{a c}$ Niall McEvoy, ${ }^{a c}$ Nikos Peltekis, ${ }^{a b}$ Jonathan N. Coleman ${ }^{a c}$ \\ and Georg S. Duesberg*ab
}

\author{
Received 20th May 2010, Accepted 5th July 2010 \\ DOI: 10.1039/c0jm01527j
}

\begin{abstract}
This paper describes the electron transfer properties of graphene nano-sheets (GNSs) immobilised on pyrolysed photoresist film (PPF) electrodes. The former are produced by the dispersion and exfoliation of graphite in dimethylformamide, and they are characterised using transmission electron microscopy, scanning electron microscopy and Raman spectroscopy. Cyclic voltammetry and electrochemical impedance spectroscopy are used to quantify the effect of the GNSs on electrochemical surface area and on electron transfer kinetics. Compelling evidence is reported in relation to the importance of edgeplane sites and defects in the promotion of electron transfer at carbon nanostructures. A novel ascorbic acid (vitamin C) sensor is presented based on the PPF/GNS system, which is effective in the range 0.4 to $6.0 \mathrm{mM}$, with a $0.12 \mathrm{mM}$ detection limit. The selectivity of the sensor is demonstrated using a commercially available vitamin $\mathrm{C}$ supplement. This is the first report of the electrochemical properties of graphene nano-sheets produced using liquid-phase exfoliation, and it will serve as an important benchmark in the development of inexpensive graphene-based electrodes with high surface area and electro-catalytic activity.
\end{abstract}

\section{Introduction}

Carbon materials are widely used in electrochemistry due to their low cost, wide potential window and electro-catalytic activity for a range of redox reactions. ${ }^{1}$ Their applications in electro-catalysis depend very much on their microstructure and surface chemistry, and the various allotropes have very different electrochemical properties. The electrochemistry of 'traditional' $\mathrm{sp}^{2}$-hybridised carbon materials such as glassy carbon and highly ordered pyrolytic graphite has been thoroughly probed, but the last decade has seen an extraordinary amount of research into the electrochemical properties of carbon nanotubes (CNTs). Besides being quasi-onedimensional electronic systems, they offer a high conductivity and surface area. Despite this, there remains a great deal of uncertainty regarding the precise mechanisms by which CNTs function as electrode materials. This arises from the use of different types of CNTs which have different electronic properties and contain various (and often unspecified) amounts of structural defects, metallic impurities and surface functionalities. ${ }^{2}$

Graphene, the newest member of the carbon family, is attracting interest as an electrode material due to its twodimensional nature. ${ }^{3,4}$ Strictly speaking, graphene is a carbon monolayer, but Geim and Novoselov ${ }^{5}$ have reported that structures composed of up to around ten graphitic sheets have electronic properties sufficiently different from bulk graphite to be classified as graphene. It should be pointed out that there is some disagreement in the literature regarding the point at which 'graphene' becomes 'graphite'. A recent article by Pumera $^{3}$

\footnotetext{
${ }^{a}$ Centre for Research on Adaptive Nanostructures and Nanodevices (CRANN),Dublin, 2, Ireland. E-mail: duesberg@tcd.ie

${ }^{b}$ School of Chemistry, Trinity College, Dublin, 2, Ireland

'School of Physics, Trinity College, Dublin, 2, Ireland

$\dagger$ Electronic supplementary information (ESI) available: Electrode design, statistical TEM analysis of GNS thickness and stability of ascorbic acid response. See DOI: 10.1039/c0jm01527j
}

recommends that structures consisting of up to one hundred layers should be thought of as graphene. The first report concerning graphene electrochemistry was published in 2008 by Shang et al. ${ }^{6}$ who used as-grown arrays of vertically aligned graphene nanosheets (GNSs) on silicon as dopamine sensors. The following year saw a number of papers reporting the modification of commercial glassy carbon electrodes (GCEs) with graphene nano-sheets produced by the chemical reduction of graphitic oxide..$^{7-16}$ The modified electrodes have been used as sensors for various analytes, and as electro-catalysts for the oxygen reduction reaction. ${ }^{9}$ They have also been combined with the redox proteins glucose oxidase ${ }^{7}$ and cytochrome $c^{10}$ in biosensors for glucose and nitric oxide, respectively. Graphene paper electrodes have been used in lithium batteries, ${ }^{17}$ and graphene oxide has been incorporated into a glucose biosensor. ${ }^{18}$ Recently, Qu et al. ${ }^{19}$ used nitrogen-doped graphene, produced using chemical vapour deposition, to promote oxygen reduction in fuel cells.

Ascorbic acid (AA) is used extensively in the food and drinks industry. The accurate determination of its concentration is of considerable importance, but the reliable sensing of AA using conventional carbon electrodes has been hindered by the large over-potentials required and electrode fouling by oxidation products. ${ }^{20}$ It is hoped that a reliable electroanalytical technique might offer greater selectivity, time efficiency and reproducibility than existing methods such as high-performance liquid chromatography, ${ }^{21}$ capillary zone electrophoresis ${ }^{22}$ and spectrophotometry. ${ }^{23}$ As a result, electrodes have been modified with carbon nanotubes ${ }^{24-26}$ and other agents such as cobalt phthalocyanine, ${ }^{27}$ ferrocene derivatives ${ }^{28}$ and ferrocene carboxylic $\operatorname{acid}^{20}$ for the detection of ascorbic acid. These efforts have met with various degrees of success.

In this paper, the development of a novel AA sensor based on pyrolysed photoresist films (PPFs) modified with graphene nano-sheets is reported. The latter are produced by the dispersion and exfoliation of graphite flakes. The fabrication process 
builds on our previous work ${ }^{29}$ describing the production of monolayer graphene. The sonication time has been extended in order to yield smaller flakes with greater electrochemical surface areas and higher electro-catalytic activity. The PPFs are made inhouse and they provide an excellent electrical contact to the sheets. They serve as a cheap and disposable alternative to the glassy carbon electrodes used in previous reports. Furthermore, being themselves less electrochemically active than GCEs, they permit the de-convolution of kinetic data and facilitate a clearer understanding of the electrochemistry of the graphene nano-sheets. The ascorbic acid sensor is shown to exhibit impressive selectivity using tests involving a commercial vitamin C supplement. A key objective of the work is the evaluation of the fundamental electron transfer characteristics of graphene nano-sheets. This is done using standard redox probes, and new insights are presented regarding the reasons for the promotion of electron transfer by the sheets.

\section{Experimental}

\section{Chemicals and instruments}

Graphite powder (product number 332461), potassium chloride (99.0\%), hexaammineruthenium(III) chloride (98\%), potassium ferrocyanide $(99.0 \%)$, potassium ferricyanide $(99.0 \%)$ and ascorbic acid $(99.0 \%)$ were purchased from Sigma-Aldrich. Rubex tablets were purchased from a local pharmacy. All solutions were prepared with water (resistivity $18.2 \mathrm{M} \Omega \mathrm{cm}$ ) from a Thermo Scientific millipore system. The $50 \mathrm{mM}, \mathrm{pH} 7$ phosphate buffer solution used was prepared by dissolving monosodium dihydrogen phosphate dihydrate $\left(\mathrm{NaH}_{2} \mathrm{PO}_{4} \cdot 2 \mathrm{H}_{2} \mathrm{O}\right)$ and disodium hydrogen phosphate dodecahydrate $\left(\mathrm{Na}_{2} \mathrm{H}-\right.$ $\mathrm{PO}_{4} \cdot 12 \mathrm{H}_{2} \mathrm{O}$ ) in water in the appropriate ratio. These two salts were purchased from Merck.

A Jeol 2100 TEM, operated at $200 \mathrm{kV}$, and a Zeiss Ultra SEM were used to obtain images of the graphene nano-sheets. Raman spectra were recorded using a Jobin-Yvon spectrometer set to an excitation wavelength of $633 \mathrm{~nm}$, with a probe size of two microns. Five spectra were obtained for each sample in order to ensure uniformity. Thicknesses of pyrolysed photoresist films were determined using a Profilometer (Dektac 6M, Veeco Instruments), and conductivities were measured using a Jandel four-point probe. A Gamry 600 potentiostat was used to perform cyclic voltammetry and electrochemical impedance spectroscopy, along with a three-electrode configuration. IJ Cambria supplied platinum wire counter electrodes (product number $\mathrm{CHI} 15$ ) and $\mathrm{Ag} / \mathrm{AgCl}$ reference electrodes (CHI111). Electrolytes were purged for twenty minutes using high-purity argon before experiments, and a blanket of argon was maintained over the solution during measurements. All electrochemical experiments were performed at room temperature $\left(18 \pm 3^{\circ} \mathrm{C}\right)$.

\section{Formation of pyrolysed photoresist films}

$\mathrm{Si} / \mathrm{SiO}_{2}$ wafers $(1.5 \times 1.5 \mathrm{~cm})$ were cleaned using acetone and isopropanol, and then blown dry using compressed air. The photoresist ( $A Z$ nLOF 2070, MicroChemicals GmbH, Ulm, Germany) was spin-coated onto these substrates at $6000 \mathrm{rpm}$ for 45 seconds, followed by baking (one minute at $100^{\circ} \mathrm{C}$ ). The samples were then annealed in a vacuum tube furnace under continuous flow of forming gas $(90 \%$ argon and $10 \%$ hydrogen). The temperature was increased at a rate of $4{ }^{\circ} \mathrm{C} \mathrm{min}^{-1}$ to $1000{ }^{\circ} \mathrm{C}$ and held at this temperature for one hour. A Profilometer was used to show a decrease of thickness upon annealing by a factor of five, resulting in films of around $20 \mathrm{~nm}$ thickness, with conductivities around $1.6 \times 10^{4} \mathrm{~S} \mathrm{~m}^{-1}$, as measured using a four-point probe.

\section{Formation of graphene nano-sheets and electrode modification procedure}

Graphite flakes were dispersed in dimethylformamide $(3.3 \mathrm{mg}$ $\mathrm{cm}^{-3}$ ) and placed in a sonic bath for 72 hours to yield graphene nano-sheets. Surviving flakes were removed by centrifuging at $750 \mathrm{rpm}$ for 45 minutes, followed by decanting the supernatant. When required, the latter was dropped $(15 \mu \mathrm{L})$ directly onto photoresist films and the solvent was allowed to dry. The resulting GNS films were clearly visible to the naked eye. The GNS-modified PPFs were incorporated into the electrochemical cell by placing the substrates in an electrode designed in-house (see ESI $\dagger$ ), resulting in a disc electrode (radius $1.5 \mathrm{~mm}$ ). The PPF and PPF/GNS surfaces were activated before measurements by cycling in background electrolyte until reproducible scans were obtained. Three or four cycles were usually found to be sufficient to achieve this. The potential ranges over which these preliminary cycles were carried out were chosen to match those of whatever measurement was to be subsequently performed.

\section{Results and discussion}

The morphology of the GNSs was investigated using transmission electron microscopy and scanning electron microscopy. Fig. 1(a) shows a typical TEM image of graphene sheets drop-coated onto a holey carbon grid. The nano-sheets are mostly sub-micron in lateral size, of various thicknesses, and in places appear to be stacked on top of each other. A statistical analysis of one hundred arbitrary flakes revealed that $\sim 90 \%$ had five layers or fewer (see ESI $†$ ). Fig. 1(b) shows a SEM image of a drop-coated GNS film on a PPF electrode surface. Some of the nano-sheets have edges which are wrinkled up away from the underlying electrode, like paint peeling off a wall. Images of the bulk graphite source from which the sheets were produced can be found in previous work, ${ }^{29}$ showing flakes at least $150 \mu \mathrm{m}$ across, so it is clear that the prolonged sonication results in a considerable decrease in flake size. The GNSs were further characterised using Raman spectroscopy, and Fig. 1(c) shows a spectrum averaged over five spots on a GNS assembly formed by drop-coating onto a $\mathrm{Si} / \mathrm{SiO}_{2}$ wafer.

Included in the figure is a spectrum recorded for the as-received graphite. The two spectra were normalized to the intensity of the $\mathrm{G}$ peak $\left(1582 \mathrm{~cm}^{-1}\right)$. The larger $\mathrm{D}$ band $\left(1330 \mathrm{~cm}^{-1}\right)$ observed for the GNSs confirms the smaller domain size, and the broader 2D peak $\left(2660 \mathrm{~cm}^{-1}\right)$ indicates the formation of thin graphitic sheets of various thicknesses, as opposed to bulk graphite. ${ }^{30}$ The diminished flake size means that the GNS material has a larger density of edge-plane sites and defects, which are known to promote electron transfer at carbon electrodes. ${ }^{30-35}$

The presence of graphene nano-sheets on the pyrolysed photoresist film surfaces was found to increase the electrochemically active surface area. The hexaammineruthenium(III) chloride redox probe was used to quantify this increase. This probe was selected for this purpose because its reaction kinetics 

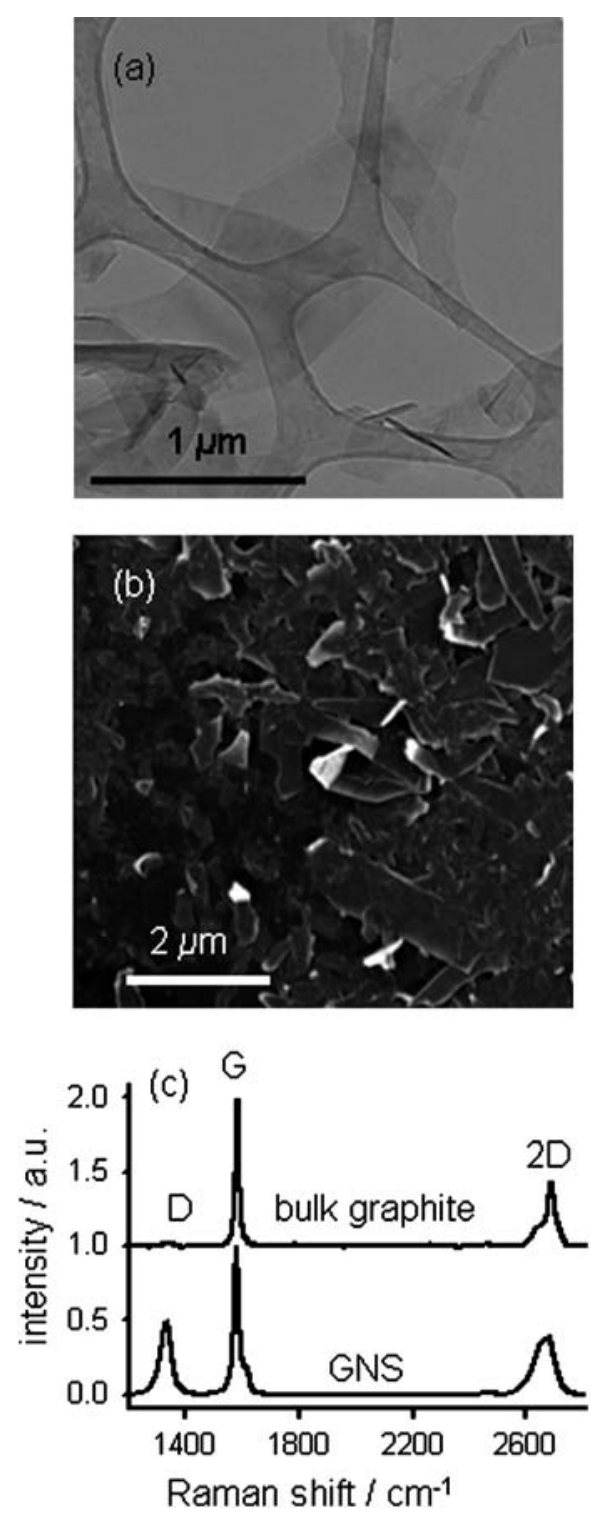

Fig. 1 (a) TEM image of graphene nano-sheets drop-coated onto a holey carbon grid. (b) SEM image of graphene nano-sheets drop-coated onto a PPF electrode. (c) Raman spectrum of graphene nano-sheets drop-coated onto a $\mathrm{Si} / \mathrm{SiO}_{2}$ wafer. For comparison, a spectrum obtained for the bulk graphite source is also shown.

are determined by the Fermi level density of states of the electrode material, ${ }^{36}$ rather than any functionalities, defects or impurities which may be present. This means that it shows reversible electrochemistry at virtually all graphitic surfaces, enabling the legitimate comparison of peak currents without the need to consider differences in reaction kinetics. Fig. 2 shows a typical comparison between bare and modified electrodes. The larger peak current for the PPF/GNS electrode is attributed to a greater electrochemically active surface area. Values for these areas were calculated using the equation

$$
i_{\mathrm{pc}}=3.01 \times 10^{5} \times n^{3 / 2} A C^{\infty} \sqrt{\alpha D \nu} .
$$

in which $i_{\mathrm{pc}}$ is the cathodic peak current, $n$ is the number of electrons involved in the redox process $(n=1$ for the $\left[\mathrm{Ru}\left(\mathrm{NH}_{3}\right)_{6}\right]^{3+/ 2+}$ couple), $A$ is the electrochemical surface area, $C^{\infty}$

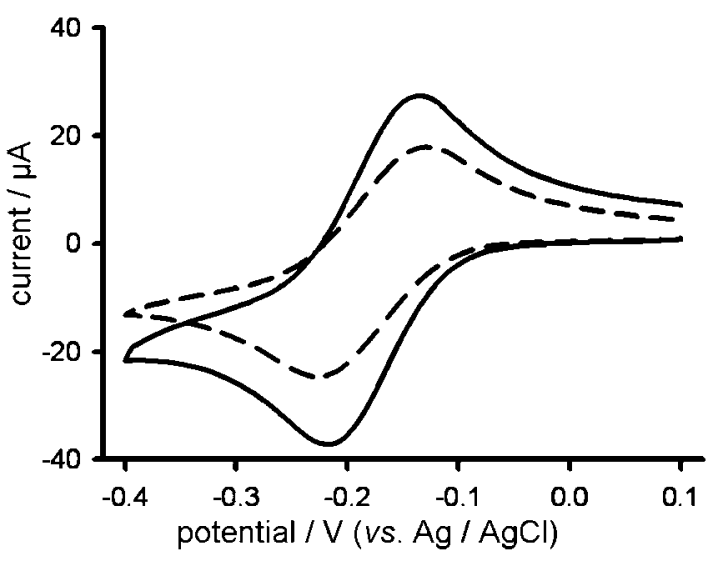

Fig. 2 Cyclic voltammograms recorded at bare (dashed) and GNSmodified (solid) PPF electrodes in $1 \mathrm{mM}$ hexaammineruthenium(III) chloride in $1 \mathrm{M}$ potassium chloride at a sweep rate of $200 \mathrm{mV} \mathrm{s}^{-1}$.

is the bulk concentration of the redox probe, $\alpha$ is the transfer coefficient (taken to be 0.5 ), $\nu$ is the voltammetric sweep rate and $D$ is the diffusion coefficient of the electro-active species, which in this case has the value of $6.2 \times 10^{-6} \mathrm{~cm}^{2} \mathrm{~s}^{-1} \cdot{ }^{37}$ Based on peak current values of 23.9 and $35.7 \mu \mathrm{A}$ obtained at a scan rate of $200 \mathrm{mV} \mathrm{s}^{-1}$, the electrochemical surface area was found to increase from 0.10 to $0.15 \mathrm{~cm}^{2}$ in the presence of the sheets. It is noted that, due to roughness, both of these values are larger than the geometric area of the electrodes $\left(0.07 \mathrm{~cm}^{2}\right)$.

The standard, quasi-reversible ferro/ferricyanide redox probe was used to elucidate the electron transfer properties of the graphene nano-sheets. Fig. 3(a) compares cyclic voltammograms obtained using bare and GNS-modified pyrolysed photoresist films. It is clear that, for this probe, the electron transfer at the bare PPF is very sluggish, with only slight waves corresponding to the redox process. This is an important point. Virtually all other studies reporting GNS electrochemistry involve the modification of commercial glassy carbon electrodes. We point out that the latter are themselves endowed with excellent electron transfer properties, and therefore the kinetic data obtained can be somewhat convoluted. We suggest that it is preferable to use relatively inert underlying electrode surfaces such as the PPFs described here. Returning to Fig. 3(a), in the presence of GNSs, a well-defined response is obtained, suggesting a dramatic enhancement in the rate of electron transfer. Fig. 3(b) shows that, for the PPF/GNS electrode, the anodic peak current is proportional to the square root of the sweep rate over the range 5 to 200 $\mathrm{mV} \mathrm{s}^{-1}$ (correlation coefficient 0.999 ). This behaviour is characteristic of diffusion-controlled electrochemical reactions. In order to provide a quantitative insight into the electron transfer properties of the graphene nano-sheets, the standard electrochemical rate constant was calculated for the ferro/ferricyanide test reaction. This was done using the Nicholson ${ }^{38}$ method, which yields values for this parameter based on voltammetric peak separations. The separation at a scan rate of $100 \mathrm{mV} \mathrm{s}^{-1}$ was found to be $120 \mathrm{mV}$, which yielded a value of $3.5 \times 10^{-3} \mathrm{~cm} \mathrm{~s}^{-1}$ for the rate constant. A more detailed description of the application of this method can be found in previous work. ${ }^{39}$ It is noted that this value is similar to that reported by Pacios et al. ${ }^{40}$ using bamboo-like carbon nanotube composite electrodes, employing the same approach. 

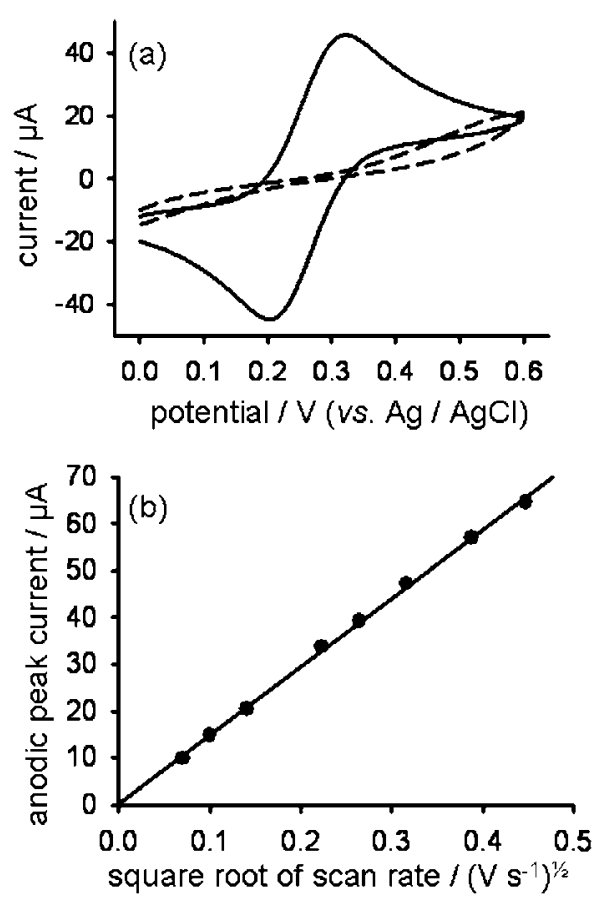

Fig. 3 (a) Cyclic voltammograms recorded at bare (dashed) and GNSmodified (solid) PPF electrodes in $1 \mathrm{mM}$ ferro/ferricyanide in $1 \mathrm{M}$ potassium chloride at a sweep rate of $100 \mathrm{mV} \mathrm{s}^{-1}$. (b) The corresponding relationship between anodic peak current and scan rate for the PPF/GNS electrode.

The enhanced electron transfer properties of the GNS-modified electrodes were also clear from electrochemical impedance spectroscopy data. Fig. 4 shows a comparison between Nyquist plots obtained using a PPF electrode in the absence and in the presence of graphene nano-sheets. In such plots, the resistance to charge transfer is indicated by the size of the semicircular region. It can be seen that the response of the bare electrode is dominated by this feature, suggesting that the reaction kinetics are under the control of electron transfer at all frequencies. However, the modified electrode gives rise to a far smaller charge transfer resistance, with a considerable linear region indicating diffusion control at moderate frequencies. The impressive properties of the GNS electrodes are attributed to their high density of edge-plane sites and defects, which have been established as key participants in the promotion of electron transfer at carbon electrodes. ${ }^{30-35}$ Despite the thinness of the flakes (see ESI $\dagger$ ), it is not envisaged that the excellent electrochemical activity of this material is due to its much-lauded electronic properties. The key to its performance is the small lateral size of the flakes created by prolonged sonication, and the resulting large proportion of edge regions. Furthermore, the material in the present work is not produced via a graphitic oxide intermediate or by a catalytic deposition, so contributions from residual oxygenated groups and metallic impurities may be ruled out. Our previous XPS studies ${ }^{29}$ have shown that graphene nano-sheet surfaces produced in this way are free from significant amounts of oxygenated functionalities.

Cyclic voltammetry was used to investigate the application of graphene nano-sheets in electrochemical sensors for ascorbic acid. Fig. 5 shows the effect of GNSs on the response of

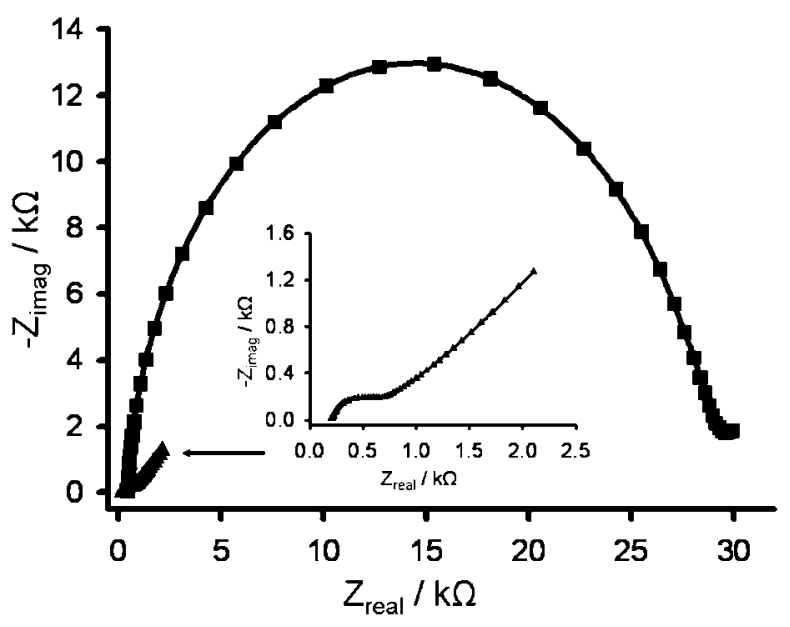

Fig. 4 Nyquist plots recorded at bare ( $\boldsymbol{\square})$ and GNS-modified ( $\mathbf{\Delta})$ electrodes in $1 \mathrm{mM}$ ferro/ferricyanide in $1 \mathrm{M}$ potassium chloride at $+0.26 \mathrm{~V}$ when an AC signal (amplitude $10 \mathrm{mV}$ ) was applied in the frequency range $10^{-1}$ to $10^{5} \mathrm{~Hz}$. For clarity, the inset shows an enlargement of the plot obtained using the modified electrode.

a pyrolysed photoresist film electrode to this analyte. While the bare electrode shows only a wave corresponding to AA oxidation, the PPF/GNS electrode gives a well-defined peak around $+0.37 \mathrm{~V}(v s . \mathrm{Ag} / \mathrm{AgCl})$. No significant decrease in peak current was observed on twenty successive measurements (see ESI $\dagger$ ), suggesting that this material is not subject to deleterious adsorption processes such as those reported for ascorbic acid on glassy carbon electrodes. ${ }^{41}$

Using a fixed sweep rate of $100 \mathrm{mV} \mathrm{s}^{-1}$, the variation of the anodic peak current, $i_{\mathrm{pa}}$, with ascorbic acid concentration was investigated. Fig. 6(a) shows the increase of $i_{\text {pa }}$ with successive additions of AA, and the resulting calibration plot in Fig. 6(b) is a straight line (correlation coefficient 0.997 ) for concentrations between 0.4 and $6.0 \mathrm{mM}$, given by the equation:

$$
i_{\mathrm{pa}}(\mu \mathrm{A})=18.5 \times C_{\mathrm{AA}}(\mathrm{mM})-7.37
$$

in which $C_{\mathrm{AA}}$ is the concentration of ascorbic acid. The limit of detection (based on $3 \sigma$ ) was found to be $0.12 \mathrm{mM}$.

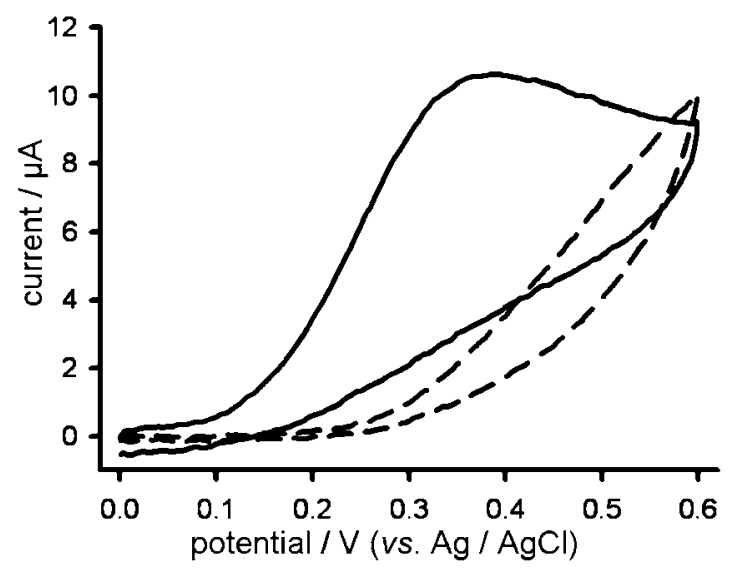

Fig. 5 Cyclic voltammograms recorded at bare (dashed) and GNSmodified (solid) PPF electrodes in $1 \mathrm{mM}$ ascorbic acid in $50 \mathrm{mM} \mathrm{pH} 7$ phosphate buffer at a sweep rate of $100 \mathrm{mV} \mathrm{s}^{-1}$. 

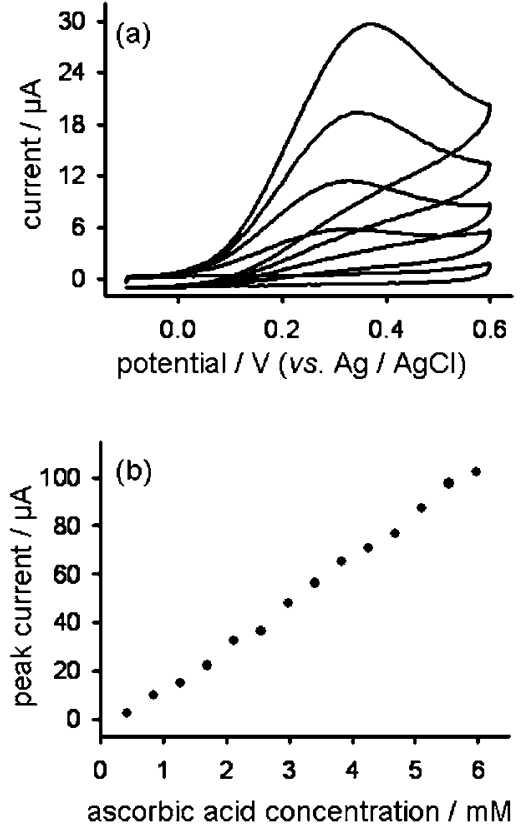

Fig. 6 (a) Cyclic voltammograms recorded at a PPF/GNS electrode in (from bottom to top) $0.0,0.8,1.2,1.6$ and $2.0 \mathrm{mM}$ ascorbic acid in $\mathrm{pH} 7$ phosphate buffer at a sweep rate of $100 \mathrm{mV} \mathrm{s}^{-1}$. (b) The resulting plot of electro-catalytic peak current against AA concentration.

The performance of the GNS-based ascorbic acid sensor was further evaluated using tests involving the commercially available vitamin $\mathrm{C}$ supplement Rubex. This product also contains a significant amount of glucose, as well as flavorings and colorants, and is therefore well-suited to such tests. According to the manufacturers, each Rubex tablet is, by mass, $29 \%$ ascorbic acid, along with $20 \%$ glucose. For these tests, $30 \mathrm{mg}$ of a tablet were dissolved in $20 \mathrm{~cm}^{3}$ of phosphate buffer $(\mathrm{pH} \mathrm{7})$ and the resulting solution was measured using a PPF/GNS electrode. Fig. 7 shows that this gave rise to a peak current of $41.0 \mu \mathrm{A}$ which, from eqn (2), corresponds to an ascorbic acid concentration of $2.61 \mathrm{mM}$, or $9.21 \mathrm{mg}$ of AA in the $20 \mathrm{~cm}^{3}$ sample. This means that the tablet was $31 \%$ ascorbic acid, which is in reasonable agreement with the provided product information.

In order to confirm the selectivity of PPF/GNS electrodes for ascorbic acid, the same sample was spiked with AA in order to produce a $2.03 \mathrm{mM}$ concentration increase. Fig. 7 shows that this caused the peak current to increase to $79.0 \mu \mathrm{A}$. Using eqn (2), this corresponds to a concentration of $4.67 \mathrm{mM}$. Subtraction of the spike gives $2.64 \mathrm{mM}$, which is very close to the originally determined concentration of $2.61 \mathrm{mM}$. This confirms that the signal is due entirely to ascorbic acid, and there are no significant contributions from glucose or other interfering species present in the sample.

\section{Conclusions}

The electrochemical properties of graphene nano-sheets (GNSs) produced by the dispersion and exfoliation of graphite have been reported. This material has been shown to consist of graphene sheets with small lateral size and high density of edge-plane sites and defects. Standard redox probes have been utilised to demonstrate the large electrochemical surface area and electro-

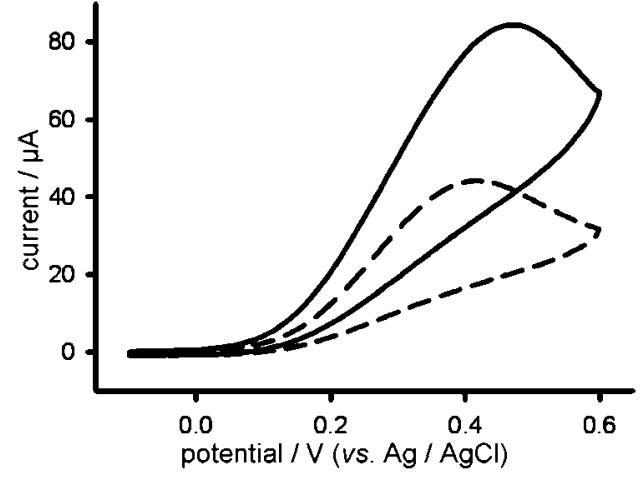

Fig. 7 Cyclic voltammograms recorded at a PPF/GNS electrode in 1.5 $\mathrm{mg} \mathrm{cm} \mathrm{cm}^{-3}$ Rubex (dashed) and the same solution spiked with $2.03 \mathrm{mM}$ ascorbic acid (solid). The supporting electrolyte was $50 \mathrm{mM} \mathrm{pH} 7$ phosphate buffer and the sweep rate was $100 \mathrm{mV} \mathrm{s}^{-1}$.

catalytic activity of the graphene nano-sheets. The unique exfoliation process is preferable to the more common chemical reduction of graphitic oxide, in that it permits the definitive identification of edge-planes as the promoters of electron transfer, an issue of continuing debate in the field of nano-carbon electrodes. A novel ascorbic acid (vitamin C) sensor based on pyrolysed photoresist films modified with GNSs has been demonstrated. This system shows no evidence of deleterious adsorption processes, and its accuracy and selectivity have been verified using a commercially available vitamin $\mathrm{C}$ supplement. These findings constitute a significant step in the development of a new class of nanostructured electrodes for sensors, fuel cells and energy conversion.

\section{Acknowledgements}

The kind assistance of Mr Jason Jensen of the Centre for Research on Adaptive Nanostructures and Nanodevices (CRANN), in relation to the printing of the custom-made electrodes employed in this work, is warmly appreciated. This work was supported by Science Foundation Ireland under the CSET scheme and the Embark initiative under the IRCSET scheme.

\section{References}

1 R. L. McCreery, Chem. Rev., 2008, 108, 2646-2687.

2 I. Dumitrescu, P. R. Unwin and J. V. Macpherson, Chem. Commun., 2009, 6886-6901.

3 M. Pumera, Chem. Rec., 2009, 9, 211-223.

4 Y. Shao, J. Wang, H. Wu, J. Liu, I. A. Aksay and J. Lin, Electroanalysis, 2010, 22, 1027-1036.

5 A. K. Geim and K. S. Novoselov, Nat. Mater., 2007, 6, 183-191.

6 N. G. Shang, P. Papakonstantinou, M. McMullan, M. Chu, A. Stamboulis, A. Potenza, S. S. Dhesi and H. Marchetto, $A d v$. Funct. Mater., 2008, 18, 3506-3514.

7 T. T. Baby, S. S. Jyothirmayee Aravind, T. Arockiadoss, R. B. Rakhi and S. Ramaprabhu, Sens. Actuators, B, 2010, 145, 71-77.

8 H. Liu, J. Gao, M. Xue, N. Zhu, M. Zhang and T. P. Cao, Langmuir, 2009, 25, 12006-12010.

9 L. Tang, Y. Wang, Y. Li, H. Feng, J. Lu and J. Li, Adv. Funct. Mater., 2009, 19, 2782-2789.

10 J.-F. Wu, M.-Q. Xu and G.-C. Zhao, Electrochem. Commun., 2010, 12, 175-177.

11 Y. Wang, Y. Wan and D. Zhang, Electrochem. Commun., 2010, 12, 187-190. 
12 C. Shan, H. Yang, J. Song, D. Han, A. Ivaska and L. Niu, Anal. Chem., 2009, 81, 2378-2382.

13 Y. Wang, Y. Li, L. Tang, J. Lu and J. Li, Electrochem. Commun., 2009, 11, 889-892.

14 M. Zhou, Y. Zhai and S. Dong, Anal. Chem., 2009, 81, 5603-5613.

15 S. Yang, D. Guo, L. Su, P. Yu, D. Li, J. Ye and L. Mao, Electrochem. Commun., 2009, 11, 1912-1915.

16 S. Alwarappan, A. Erdem, C. Liu and C.-Z. Li, J. Phys. Chem. C, 2009, 113, 8853-8857.

17 C. Wang, D. Li, C. O. Too and G. G. Wallace, Chem. Mater., 2009, 21, 2604-2606.

18 X. Kang, J. Wang, H. Wu, I. A. Aksay, J. Liu and Y. Lin, Biosens. Bioelectron., 2009, 25, 901-905.

19 L. Qu, Y. Liu, J.-B. Baek and L. Dai, ACS Nano, 2010, 4, 1321-1326.

20 J.-B. Raoof, R. Ojani and A. Kiani, J. Electroanal. Chem., 2001, 515, $45-51$.

21 J. A. Albhecht and H. W. Schafer, J. Liq. Chromatogr. Relat. Technol., 1990, 13, 2633-2641.

22 M. Chiari, M. Nesi, G. Carrea and P. G. Righetti, J. Chromatogr., A, 1993, 645, 197-200.

23 S. L. C. Ferreira, M. L. S. F. Bandeira, V. A. Lemos, H. C. Dos Santos, A. C. S. Costa and D. S. De Jesus, Fresenius J. Anal. Chem., 1997, 357, 1174-1178.

24 H. Luo, Z. Shi, N. Li, Z. Gu and Q. Zhuang, Anal. Chem., 2001, 73, 915-920.

25 J. Wang, M. Li, Z. Shi, N. Li and Z. Gu, Electroanalysis, 2002, 14 225-230.

26 M. D. Rubianes and G. A. Rivas, Electrochem. Commun., 2003, 5, 689-694.

27 S. A. Wring, J. P. Hart and B. J. Birch, Anal. Chim. Acta, 1990, 229, 63-70.

28 R. Ojani and M. H. Pournaghi-Azar, Talanta, 1995, 42, 1839-1848.
29 Y. Hernandez, V. Nicolosi, M. Lotya, F. M. Blighe, Z. Sun, S. De, I. T. McGovern, B. Holland, M. Byrne, Y. K. Gun'ko, J. J. Boland, P. Niraj, G. S. Duesberg, S. Krishnamurthy, R. Goodhue, J. Hutchison, V. Scardaci, A. C. Ferrari and J. N. Coleman, Nat. Nanotechnol., 2008, 3, 563-568.

30 A. C. Ferrari, J. C. Meyer, V. Scardaci, C. Casiraghi, M. Lazzeri, F. Mauri, S. Piscanec, D. Jiang, K. S. Novoselov, S. Roth and A. K. Geim, Phys. Rev. Lett., 2006, 97, 187401.

31 A. Chou, T. Böcking, N. K. Singh and J. J. Gooding, Chem. Commun., 2005, 842-844.

32 A. F. Holloway, K. Toghill, G. G. Wildgoose, R. G. Compton, M. A. H. Ward, G. Tobias, S. A. Llewellyn, B. N. Ballesteros, M. L. H. Green and A. Crossley, J. Phys. Chem. C, 2008, 112, 10389-10397.

33 E. C. Landis, K. L. Klein, A. Liao, E. Pop, D. K. Hensley, A. V. Melechko and R. J. Hamers, Chem. Mater., 2010, 22, 23572366.

34 R. J. Rice and R. L. McCreery, Anal. Chem., 1989, 61, 1637-1641.

35 R. R. Moore, C. E. Banks and R. G. Compton, Analyst, 2004, 129, $755-758$.

36 P. Chen, M. A. Fryling and R. L. McCreery, Anal. Chem., 1995, 67, 3115-3122.

37 F. G. Chevallier, N. Fietkau, J. del Campo, R. Mas, F. X. Munoz, L. Jiang, T. G. J. Jones and R. G. Compton, J. Electroanal. Chem., 2006, 596, 25-32.

38 R. S. Nicholson, Anal. Chem., 1965, 37, 1351-1355.

39 M. E. G. Lyons and G. P. Keeley, Sensors, 2006, 6, 1791-1826.

40 M. Pacios, M. del Valle, J. Bartroli and M. J. Esplandiu, J. Electroanal. Chem., 2008, 619, 117-124.

41 R. G. Compton and F.-M. Matysik, Electroanalysis, 1996, 8, 218 222. 